\title{
A robust approach for tree segmentation in deciduous forests using small-footprint airborne LiDAR data
}

\author{
Hamid Hamraz ${ }^{a^{*}}$, Marco A. Contreras ${ }^{\mathrm{b}}$, Jun Zhang ${ }^{\mathrm{a}}$ \\ a: Department of Computer Science, b: Department of Forestry \\ University of Kentucky, Lexington, KY 40506, USA \\ hhamraz@cs.uky.edu, marco.contreras@uky.edu, jzhang@cs.uky.edu \\ *Corresponding Author: $\quad$ hhamraz@ cs.uky.edu +1 (859) 4891261
}

\begin{abstract}
.
This paper presents a non-parametric approach for segmenting trees from airborne LiDAR data in deciduous forests. Based on the LiDAR point cloud, the approach collects crown information such as steepness and height on-the-fly to delineate crown boundaries, and most importantly, does not require a priori assumptions of crown shape and size. The approach segments trees iteratively starting from the tallest within a given area to the smallest until all trees have been segmented. To evaluate its performance, the approach was applied to the University of Kentucky Robinson Forest, a deciduous closed-canopy forest with complex terrain and vegetation conditions. The approach identified $94 \%$ of dominant and co-dominant trees with a false detection rate of $13 \%$. About $62 \%$ of intermediate, overtopped, and dead trees were also detected with a false detection rate of $15 \%$. The overall segmentation accuracy was $77 \%$. Correlations of the segmentation scores of the proposed approach with local terrain and stand metrics was not significant, which is likely an indication of the robustness of the approach as results are not sensitive to the differences in terrain and stand structures.
\end{abstract}

Keywords: crown delineation, remote sensing, tree-level forest data, remote forest inventory, tree detection evaluation. 


\section{Introduction}

Traditionally, decision making in forest management has been based on stand attribute information collected using stand examinations and topographic surveys. Because field-based inventory data are expensive and labor-intensive to acquire, sampling intensity of field inventory is typically limited, providing rough estimates of stand attributes while ignoring large variability in terrain and vegetation within stands (Shiver and Borders, 1996). In the last two decades, light detection and ranging (LiDAR) technology has brought drastic changes to forest data acquisition and management by providing inventory data at unprecedented spatial and temporal resolutions (Ackermann, 1999; Maltamo et al., 2014; Shao and Reynolds, 2006; Wehr and Lohr, 1999). However, in order to obtain accurate tree level attributes such as crown width and tree height as well as derivative estimates (i.e., diameter at breast height (DBH), volume, and biomass), accurate and automated tree segmentation approaches are required (Schardt et al., 2002).

Numerous methods have been developed to segment individual trees from LiDAR data. Earlier methods used pre-processed data in the form of raster digital surface models (DSMs) or canopy height models (CHMs) and more recent methods directly used the LiDAR point clouds (Hyyppä et al., 2001; Persson et al., 2002; Wang et al., 2008). Regardless of input, existing tree segmentation methods can be categorized as parametric and non-parametric. In general, parametric methods fit 3D shape models (Holmgren et al., 2010) or perform multi-stage filtering (Falkowski et al., 2006; Pitkänen et al., 2004; Wolf and Heipke, 2007), where the filtering kernel functions or the shape models are assumed to adequately estimate the geometric shapes representing the tree crowns. The parameters defining these functions/models are set manually based on typical tree crown shapes and sizes obtained previously from field sampling. A recent multi-stage filtering method (Jing et al., 2012) applies a series of morphological opening operations (Serra, 1986; Soille, 2003) to determine the dominant sizes of the tree crowns, allowing the parameters of the filter kernel to be set automatically for each stage. Although this method avoid manually setting parameters, selecting appropriate kernel function and combining the result of different stages is non-trivial, especially in natural forests with highly variable crown shapes and sizes.

Non-parametric methods identify local maxima (LMXs), assumed as the tree apexes, or use local minima (LMs) to find tree crown boundaries. LMX-based methods search for tree 
apexes within a neighborhood window and then perform a variety of region-growing or clustering routines to delineate tree crowns (Alizadeh Khameneh, 2013; Hyyppä et al., 2001; Morsdorf et al., 2004; Persson et al., 2002; Véga and Durrieu, 2011). Determining the size of the neighborhood window to search for the tree apexes is non-trivial and can easily result in missing apexes or identifying false trees. A widely used approach is to adaptively size the window based on the tree height using site-specific regression models (Chen et al., 2006; Popescu and Wynne, 2004; Popescu et al., 2002). However, this approach works well only when trees are homogeneously shaped where an accurate crown width model based on trees height only can be created (Pitkänen et al., 2004). More recent methods perform multi-stage non-parametric segmentation (Véga and Durrieu, 2011; Véga et al., 2014) where a variety of window sizes are used to create segmentation maps at different scales. The results of the different stages are then incorporated according to a scoring system based on different properties of an ideal crown shape. Li et al. (2012) resolved the problem of correctly identifying LMX by assuming the highest nonclustered LiDAR point represented the apex of the tallest tree, however, the clustering method was also considering only the vegetation height.

LM-based methods typically use watershed segmentation routines (Beucher and Lantuéjoul, 1979; Vincent and Soille, 1991) to detect crown boundaries and perform subsequent valley following routines to find the area representing individual tree crowns (Gougeon, 1995; Leckie et al., 2003). In general, watershed segmentation is prone to under/over-segmentation due to differences in tree heights and natural variability of vegetation within tree crowns. To overcome this problem, studies use marker-controlled watershed segmentation routines (Dougherty et al., 2003), where the basic idea is to mark the trees and guide the watershed procedure to only delineate those marked trees. Marking manually (Chen et al., 2006) is impractical for large-scale data. Automated approaches have generally marked the tree apexes by performing morphological image analysis (Kwak et al., 2007). Similar to LMX-based methods, these automated approaches are prone to missing tree apexes or identifying false ones, especially when trees are not homogeneously shaped and sized. Several methods have used a combination of apex identification (LMX-based) and watershed segmentations (LM-based) to perform crown delineation and thus improve tree detection rates (Chen et al., 2006; Hu et al., 2014; Jing et al., 2012; Kwak et al., 2007). 
Existing tree segmentation methods have mostly focused on conifer forests or forests with relatively open canopy, where assumptions about size and shape of tree crowns and/or spacing among trees are made (Heurich, 2008; Kaartinen et al., 2012). These assumptions make the methods forest type specific and not easily applicable to forests with different conditions (Vauhkonen et al., 2011). Deciduous forests present significantly more complex vegetation conditions due to large variation in tree shapes and sizes, larger number of species and denser canopy, where individual trees are much harder to detect (Heurich, 2008; Jing et al., 2012; Koch et al., 2006; Véga et al., 2014). Studies report that performance of previous methods varies drastically from $50 \%$ to over $90 \%$ of tree detection accuracy depending on the forest conditions and types, species distribution, and stand structure (Kaartinen et al., 2012; Vauhkonen et al., 2011). These results suggest that there is no universally superior method and that these methods are custom designed for specific vegetation conditions, which evidence the need to develop general approaches that can be applied to multiple forest types while ensuring robust tree detection results.

In this paper, we present a robust and novel approach for segmenting trees within smallfootprint LiDAR data in deciduous forests with complex terrain and vegetation conditions. The method is non-parametric and delineates individual tree crowns based on only the local information, crown shape and height of the vegetation, and does not require a priori knowledge of either stand structure or typical tree attributes. A major improvement of our approach, compared with existing approaches, is the dynamic capture of local information about crown shape and its use to enhance crown delineation.

\section{Tree segmentation approach}

The main inputs of the tree segmentation approach are the LiDAR point cloud and the LiDARderived DEM. Independent of the point density, LiDAR point clouds have variable, small-scale point spacing resulting from scan patterns (e.g., zig-zag) and flight line overlap. Thus, a preprocessing routine is applied to homogenize point spacing. This routine creates a grid with resolution equal to the average nominal post spacing (NPS) and filters the LiDAR point cloud by selecting the highest elevation LiDAR point within each grid cell, hereafter called LiDAR surface points (LSPs). Using the LiDAR-derived DEM, heights above ground are calculated for all LSPs. Those LSPs below a minimum height, set here as 5 meter, are removed from further 
analysis. Based on the vegetation structure (stem density and variability in tree heights), this creates several gaps with no vegetation in the remaining LSP dataset, which is utilized later in the analysis. The last pre-processing step smooths LSPs to reduce small variation in vegetation elevation within tree crowns while maintaining important vegetation patterns. A Gaussian smoothing filter with standard deviation equal to the NPS and a radius of $3 \times$ NPS was used.

After the pre-processing steps, the tree segmentation approach consists of the following routines: 1) locate the global maximum elevation (GMX) amongst LSPs, which is assumed to represent the apex of the tallest tree within a given area, 2) generate vertical profiles originating from the GMX location and expanding outwards, 3) identify the individual LSP along the profile that likely represents the crown boundary using between-tree gap identification and LM identification for each profile, 4) create a convex hull of boundary points, which delineates the tree crown, and 5) cluster all LSPs encompassed within the convex hull and assign them as the current tallest tree crown. This process is applied iteratively until all LSPs have been clustered into tree crowns. Clusters representing crowns with diameter below a minimum detectable crown width (MDCW), set here as $1.5 \mathrm{~m}$, are considered noise. Figure 1 shows the flowchart of the tree segmentation approach and Figure 2 shows an example of the application of the five routines within the approach. 


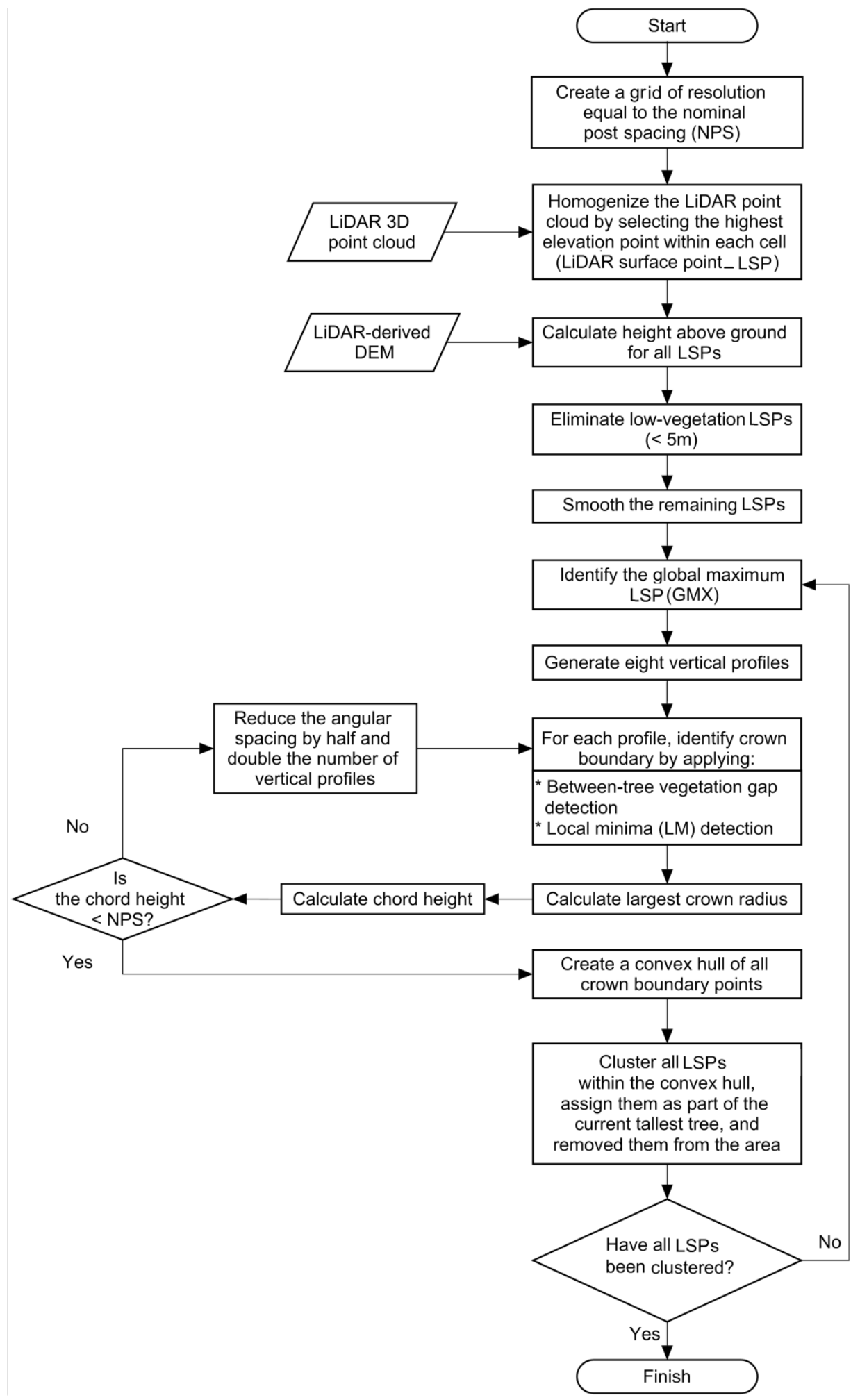

Figure 1. Flowchart of the tree segmentation approach used to identify tree locations and delineate tree crowns. 

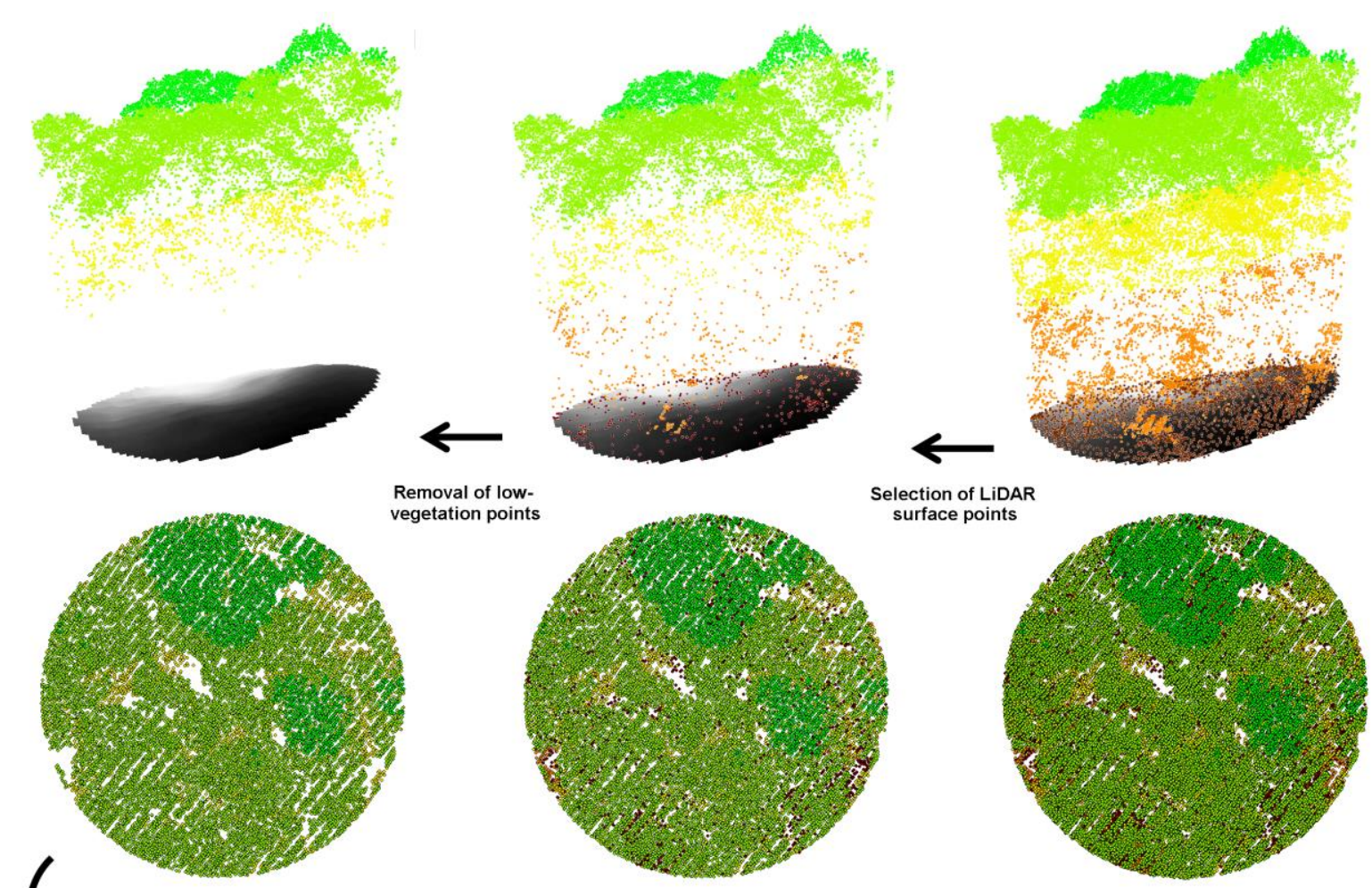

vegetation
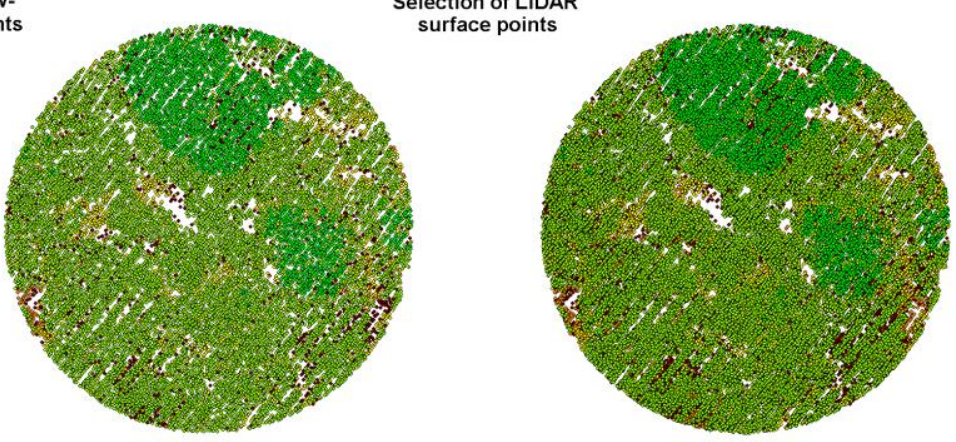

Global maximum identification and

generation of vertical profiles
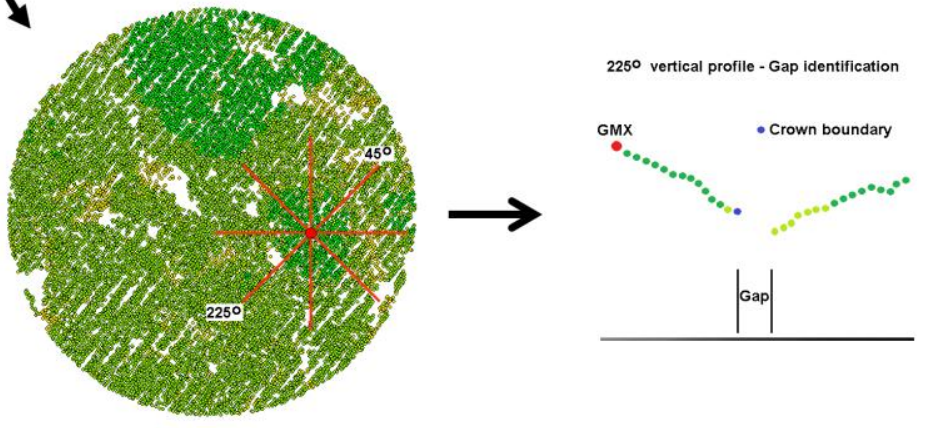

450 vertical profile - Local minima identification
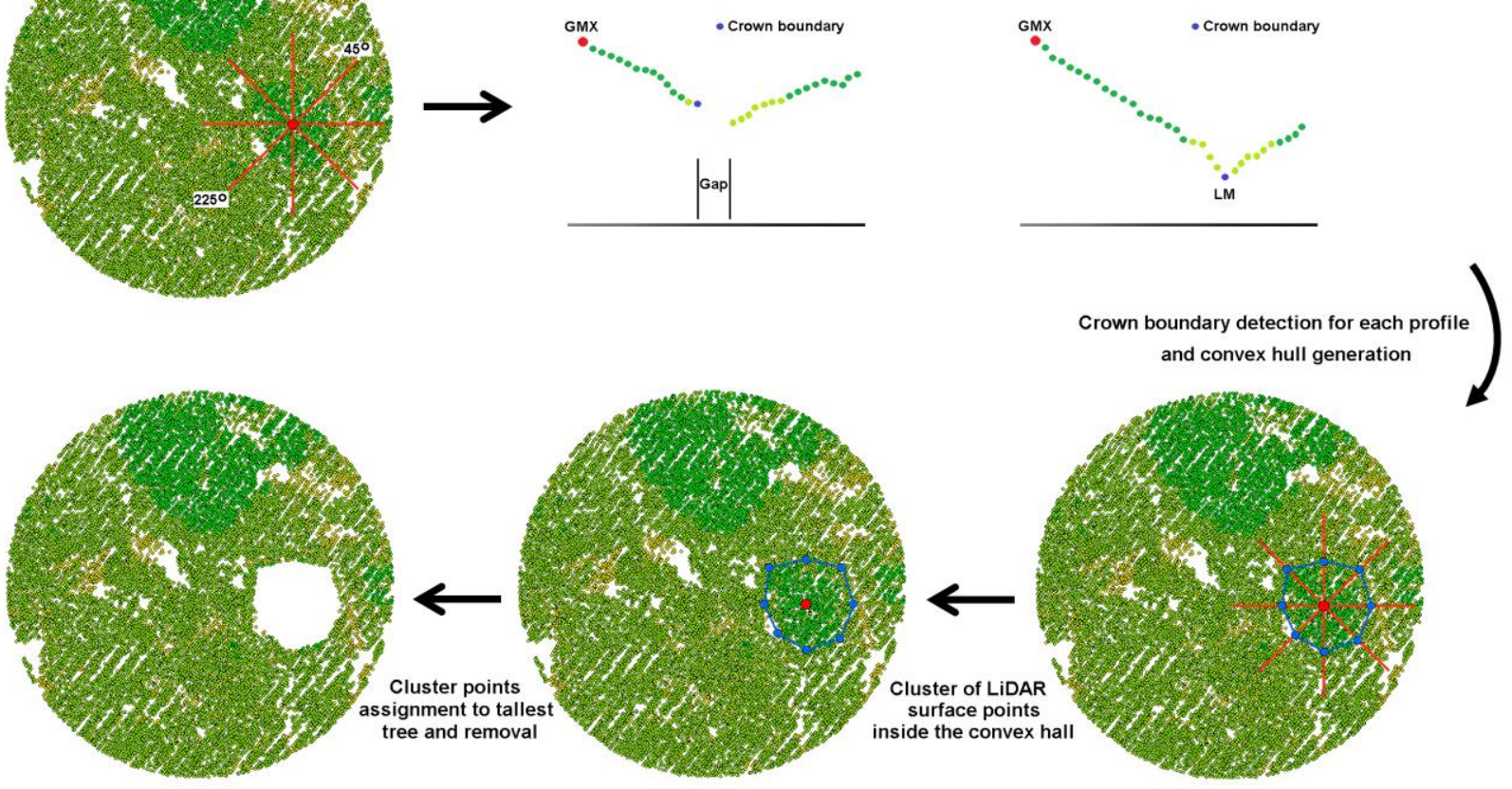

Figure 2. Illustration of the preprocessing steps and the five routines within the tree segmentation approach. 
The most critical and non-trivial routines of the tree segmentation approach are the generation of an appropriate number of profiles and the identification of crown boundary points to accurately delineate tree crowns. The procedures developed for these two routines form the basis for this novel tree segmentation approach.

\subsection{Profile generation}

After identifying the GMX within a given area, vertical profiles originating from it and expanding a maximum horizontal distance, set here to $15.24 \mathrm{~m}$ (50 feet), are generated. The number of profiles required to smoothly represent tree crowns is determined dynamically based on LiDAR-detected crown radii. The procedure starts with eight uniformly spaced profiles (every $45^{\circ}$ ). After the crown boundary and thus radius is determined for each profile (explained below), the maximum crown radius $(r)$ is used to determine the chord height $(x)$ between two maximum crown radius profiles separated by the angular spacing $(\varphi)$ (Figure 3 ) as follows:

$$
x=r(1-\cos (\varphi / 2))
$$

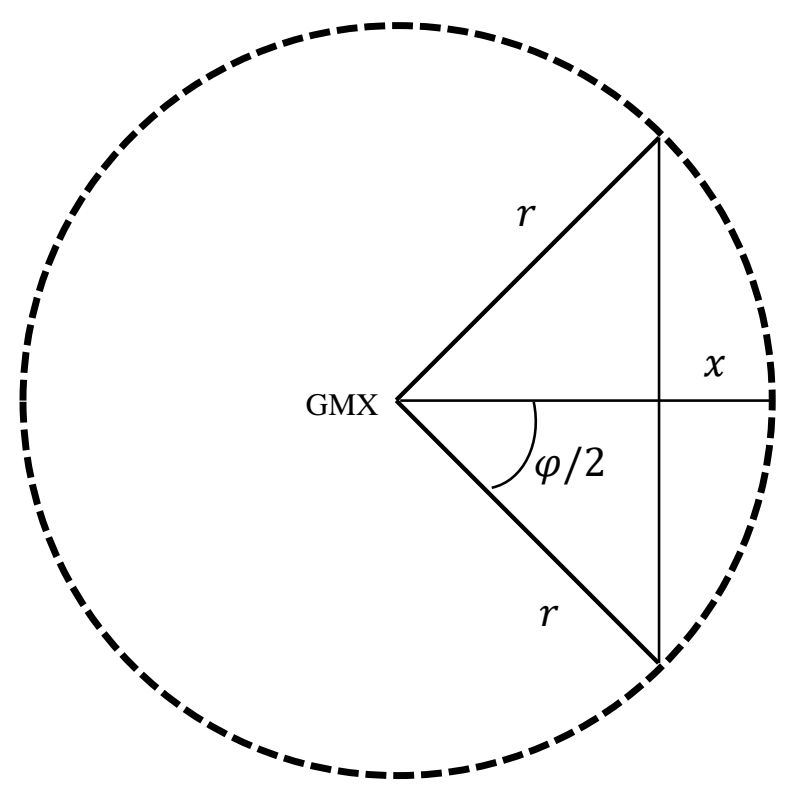

Figure 3. Diagram illustrating the calculation of the chord height $(x)$ formed by two profiles of maximum crown radius $(r)$ separated by the angular spacing $(\varphi)$. 
If the chord height is larger than NPS, the angular spacing is reduced by half and the number of profiles is doubled. The new chord height is calculated again based on the updated maximum crown radius and the new profile angular spacing. Doubling the number of profiles continues iteratively until the chord height is smaller than NPS. By using the maximum LiDARdetected crown radius, the procedure ensures a sufficiently large number of profiles and thus a smooth delineation of the tree crown.

The width of each profile was set to $2 \times$ NPS to ensure a sufficient number of LSPs representing vegetation characteristics. Profiles are then analyzed vertically in two dimensions using horizontal distance from the GMX and the elevation associated with each LSP.

\subsection{Crown boundary identification}

After generating a vertical profile and identifying all LSPs along it, two sub-routines are applied to identify the crown boundary. The first sub-routine identifies inter-tree crown gaps via statistical analysis of the distribution of horizontal distances between consecutive points along the profile. Thereafter, the second sub-routine inspects LM points as potential crown boundaries based on the median slope of points within two windows expanding both directions from each LM location.

\subsubsection{Identifying inter-tree gaps}

This sub-routine identifies relatively large horizontal distance between any two consecutive LSPs along the profile, which are assumed to represent vegetation gaps between adjacent tree crowns. For this purpose, the Tukey statistical outlier detection method is used (McGill et al., 1978). Horizontal distances between consecutive points along the profile follow a Poisson distribution. When transformed to their square root, the resulting distribution can reasonably approximate a normal distribution (Thacker and Bromiley, 2001), which is more appropriate for the Tukey method. To be conservative, square root distance values larger than six times the interquartile range from the third quartile is considered inter-tree gaps. After gaps are identified, only the LSPs between the GMX and the first gap remain for further analysis and the LSPs located beyond the first gap are removed from the profile. At this point, it is assumed that the remaining LSPs represent either the crown of the current tallest tree or the crowns of multiple adjacent trees, growing close together with overlapping crowns. 


\subsubsection{Identifying LM points as crown boundary}

Starting from the GMX, this sub-routine identifies LM points defined as those with elevations lower than their two adjacent neighbors. Once an LM point is found, the sub-routine determines whether it represents the crown boundary or natural variation of vegetation height within the crown. For this purpose, two windows expanding on both sides of the LM are created. The left window considers all LSPs from the GMX to the LM. The size of the right window is estimated based on the: i) steepness of consecutive points within a distance equal to MDCW on the right of the LM, and ii) crown radii of two hypothetical trees of equal height crowns of which represented by two distinctly different shapes (a sphere and a narrow cone).

The steepness of LSPs on the right of the LM $\left(S_{\text {right }}\right)$ is calculated as the median (in degrees) of absolute slopes between consecutive points $(i, i+1)$ within MDCW meters from the $\operatorname{LM}\left(w_{M D C W}\right)$ :

$$
S_{\text {right }}=\tan ^{-1}\left(\text { median }\left[\mid \text { slope }_{i, i+1} \mid ; i, i+1 \epsilon w_{M D C W}\right]\right)
$$

If the LM is in fact the crown boundary, the LSPs within $w_{M D C W}$ partially represent the crown of an overlapping and shorter tree with a steepness that is approximated by $S_{\text {right }}$. The value of $S_{\text {right }}$ should range between the steepness of a sphere-shaped crown and the steepness of a narrow cone-shaped crown (two ends of the spectrum). As the height of the adjacent tree $\left(h_{a d}\right)$ is between the heights of the GMX and the LM point, its height is reasonably approximated by the average of the GMX and the LM heights.

The steepness of a narrow cone-shaped crown can be expressed as $90^{\circ}-\epsilon$, where $\epsilon$ (set here as $\left.5^{\circ}\right)$ indicates a small deviation from vertical. The cone-shaped crown radius $\left(\mathrm{cr}_{\mathrm{c}}\right)$ can then be calculated as follows:

$$
c r_{c}=\frac{h_{a d} \times C L_{c}}{\tan \left(90^{\circ}-\varepsilon\right)} \times O_{c}
$$

where, $C L_{c}$ is the crown ratio, and $O_{c}$ indicates the crown radius reduction due to the overlap assuming the narrow cone-shaped tree is situated in a dense stand.

On the other hand, the slope of a sphere-shaped crown ranges from $0^{\circ}$ to $90^{\circ}$ with the steepness (expected value) of $32.7^{\circ}$ (see Appendix A). Its crown radius $\left(c r_{S}\right)$ can be calculated as follows: 


$$
c r_{s}=\frac{h_{a d} \times C L_{s}}{2} \times O_{s}
$$

where, $C L_{S}$ and $O_{s}$ indicate the crown ratio and the crown radius reduction due to the overlap within a dense stand for the sphere-shaped tree.

Then, the size of the right window $\left(w_{r d}\right)$ is calculated by interpolating $c r_{c}$ and $c r_{s}$ with respect to $S_{\text {right }}$ (which should be between $32.7^{\circ}$ and $90^{\circ}-\epsilon$ ):

$$
w_{r d}=c r_{c}\left(1-\frac{(90-\varepsilon)-S_{\text {right }}}{(90-\varepsilon)-32.7}\right)+c r_{s}\left(\frac{(90-\varepsilon)-S_{\text {right }}}{(90-\varepsilon)-32.7}\right)
$$

Lastly, after determining both window sizes on either side of the LM, the median of slopes between consecutive LSPs of each window is calculated. If the median slope of the leftside window is negative (downwards from the apex to the crown boundary) and the median slope of the right-side window is positive (upwards from the crown boundary toward the apex of the adjacent tree crown), then the LM is considered a boundary point. Otherwise, the current LM is considered to represent natural variation of vegetation height within the current tallest tree crown and the next LM farther from the GMX along the profile is evaluated. If none of the LMs found meet the crown boundary criterion then the last LSP is considered as the crown boundary.

Crown ratio is highly variable among individual trees and species dependent with values typically varying between 0.4 and 0.8 (Randolph, 2010). The crown ratio of a narrow coneshaped tree tends to be larger than that of a sphere-shaped one (Kim et al., 2009). So, for the purpose of illustrating the application of our approach, we used 0.8 and 0.7 for $\mathrm{CL}_{c}$ andCL , respectively. Similarly, crown radius reduction due to overlap is highly variable with a value of about 0.5 for a really dense stand. The radius of a narrow cone-shaped tree tends to be reduced less than of a sphere-shaped tree because the crown of a narrow cone-shaped tree is quite compact from the sides. So, we used two thirds for $\mathrm{O}_{\mathrm{c}}$ and one third for $\mathrm{O}_{\mathrm{s}}$. Although the constant values set here can affect the final size determined for the right window (Equation 5), the sign of the median slope would be the same as long as the size is within a reasonable range. Still possible in practice, an excessively narrow window might result in erroneously flipping the sign of the median slope and an LM representing natural vegetation height within the crown to be misidentified as the crown boundary and vice versa. However, when considering the multiple 
profiles generated for each GMX, the effect of a single window size on the ability to delineate tree crown is minimal.

Both sub-routines, to identify inter-tree gaps and crown boundaries respectively, are completely based on the 3D positions of LSPs along a profile. This avoids prior assumptions of tree crown shapes and dimensions, which makes the approach a robust method that can be applied to different vegetation types.

\section{Approach Application}

\subsection{Study site and field data}

The tree segmentation approach application was conducted at the University of Kentucky's Robinson Forest (RF, Lat. 37.4611, Long. -83.1555) located in the rugged eastern section of the Cumberland Plateau region of southeastern Kentucky in Breathitt, Perry, and Knott counties (Figure 4). The terrain across RF is characterized by a branching drainage pattern, creating narrow ridges with sandstone and siltstone rock formations, curving valleys and benched slopes. The slopes are dissected with many intermittent streams (Carpenter and Rumsey, 1976) and are moderately steep ranging from 10 to over $100 \%$ facings predominately northwest and south east, and elevations ranging from 252 to 503 meters above sea level. Vegetation is composed of a diverse contiguous mixed mesophytic forest made up of approximately 80 tree species with northern red oak (Quercus rubra), white oak (Quercus alba), yellow-poplar (Liriodendron tulipifera), American beech (Fagus grandifolia), eastern hemlock (Tsuga canadensis) and sugar maple (Acer saccharum) as dominant and codominant species. Understory species include eastern redbud (Cercis canadensis), flowering dogwood (Cornus florida), spicebush (Lindera benzoin), pawpaw (Asimina triloba), umbrella magnolia (Magnolia tripetala), and bigleaf magnolia (Magnolia macrophylla) (Carpenter and Rumsey, 1976; Overstreet, 1984). Average canopy cover across Robinson Forest is about $93 \%$ with small opening scattered throughout. Most areas exceed 97\% canopy cover and recently harvested areas have an average cover as low as $63 \%$. After being extensively logged in the 1920's, Robinson Forest is considered second growth forest ranging from 80-100 years old, and is now protected from commercial logging and mining activities, typical of the area (Department of Forestry, 2007). 


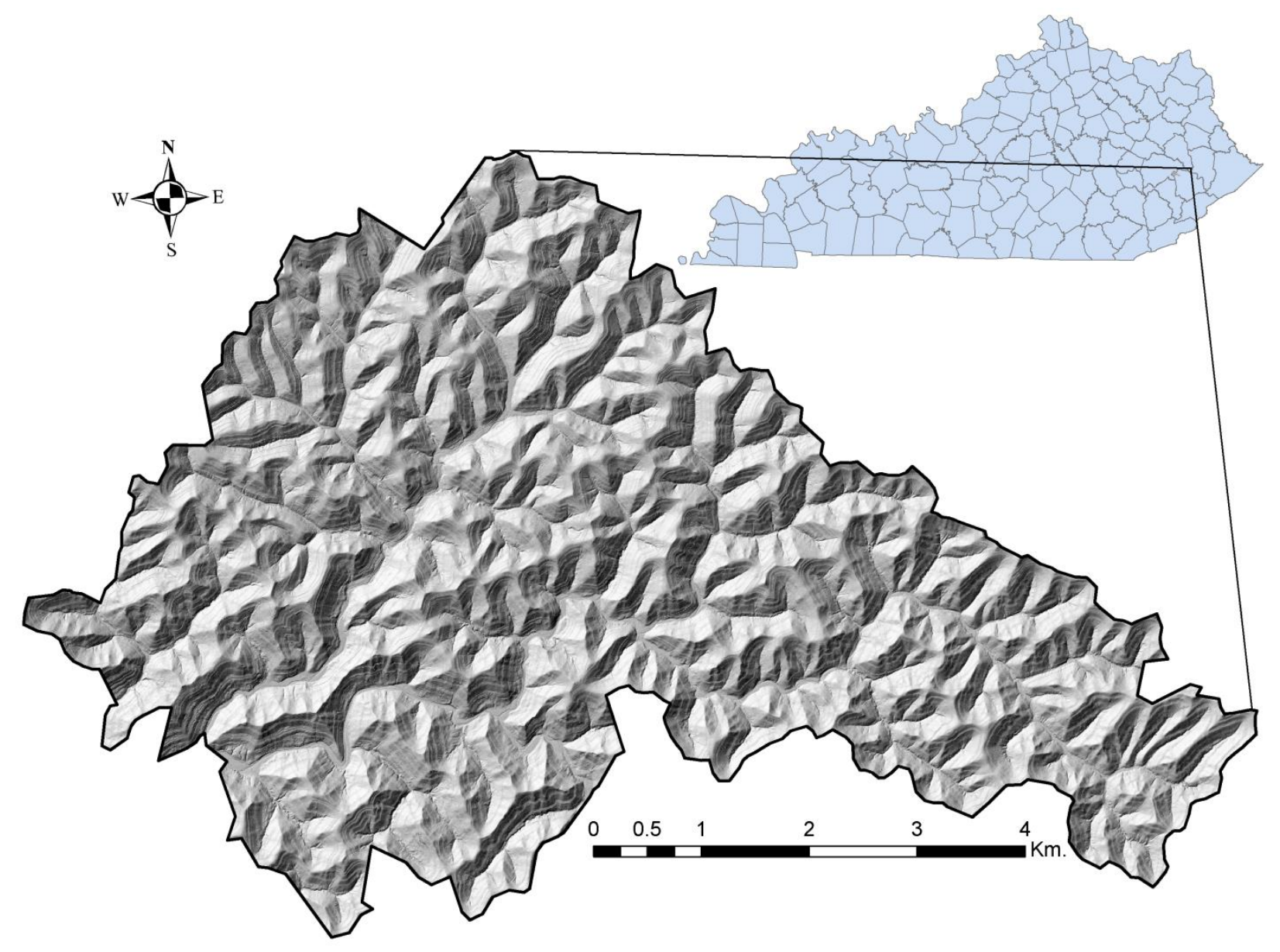

Figure 4. Terrain relief map of the University of Kentucky Robinson Forest and its general location within Kentucky, USA.

Within the Clemons Fork watershed (which covers an area of about 1,500 ha), $1.2 \times 1.2$ meter plywood boards, painted white to increase reflectance were installed prior the acquisition of LiDAR data at 103 existing circular permanent plots each with an area of 0.1 ac ( 0.04 ha). Boards were installed, leveled with their centers placed at the exact location of the plot rebar markers, with the purpose of correctly geo-referencing the exact location of plot centers. Within each plot, DBH $(\mathrm{cm})$, tree height $(\mathrm{m})$, species, crown class (dominant, co-dominant, intermediate, overtopped), tree status (live, dead), and stem class (single, multiple) were recorded for all trees with $\mathrm{DBH}>$ than $12.5 \mathrm{~cm}$. In addition, horizontal distance and azimuth from plot center to the face of each tree at breast height were collected to create a stem map. Site variables including slope, aspect, and slope position were also recorded for each plot. 


\subsection{LiDAR data}

We combined two LiDAR datasets covering the study area, collected with the same LiDAR system by the same vendor. One dataset was low density $\left(\sim 1.5 \mathrm{pt} / \mathrm{m}^{2}\right)$ collected in the spring of 2013 during leaf-off season for the purpose of acquiring terrain information, as a part of a statewide elevation data acquiring program from the Kentucky Division of Geographic Information. The second dataset was high density $\left(\sim 25 \mathrm{pt} / \mathrm{m}^{2}\right)$ acquired in the summer of 2013 during leaf-on season for the purpose of collecting detailed vegetation information and ordered by the University of Kentucky Department of Forestry. The parameters of the LiDAR system and flight for both datasets are presented in Table 1. The vendor processed both raw LiDAR datasets using the TerraScan software (Terrasolid Ltd., 2012) to classify LiDAR points into ground and nonground points. The LASTools (Isenburg, 2011) extension in ArcMap 10.2 was used to create a single LAS dataset file containing both LiDAR datasets, which was then filtered to include ground points only and create a 1-meter resolution DEM using the natural neighbor as the fill void method and the average as the interpolation method.

Table 1. LiDAR data acquisition parameters of both datasets collected over Robinson Forest.

\begin{tabular}{lll}
\hline & Leaf-Off Dataset & Leaf-On Dataset \\
\hline Date of Acquisition & April 23, 2013 & May 28- 30, 2013 \\
LiDAR System & Leica ALS60 & Leica ALS60 \\
Average Flight Elevation above Ground & $3,096 \mathrm{~m}$ & $196 \mathrm{~m}$ \\
Average Flight Speed & $105 \mathrm{knots}$ & $105 \mathrm{knots}$ \\
Pulse Repetition Rate & $200 \mathrm{KHz}$ & $200 \mathrm{KHz}$ \\
Field of View & $40^{\circ}$ & $40^{\circ}$ \\
Swath Width & $2,253.7 \mathrm{~m}$ & $142.7 \mathrm{~m}$ \\
Usable Center Portion of Swath & $90 \%$ & $95 \%$ \\
Swath Overlap & $50 \%$ & $50 \%$ \\
Average Footprint & $0.6 \mathrm{~m}$ & $0.15 \mathrm{~m}$ \\
\hline Nominal Post Spacing & $0.8 \mathrm{~m}$ & $0.2 \mathrm{~m}$ \\
\hline
\end{tabular}

\subsection{Performance evaluation}

To evaluate the performance of the tree segmentation approach, we compared the location of trees in the stem map created from field collected data with the location of LiDAR-derived tree locations. As stump locations seldom coincide with the location of the crown apexes (LiDARderived tree locations) due to leaning and irregular crown shape, the exact coordinates from the 
stem map were not used in the evaluation. Instead, we improved the tree detection evaluation procedure used by Kaartinen et al. (2012). A LiDAR-derived tree location matches with a stem map location if: i) the angle between the vertical projection of the $3 \mathrm{D}$ coordinates of the stump location and the 3D coordinates of the LiDAR-detected apex is within a given leaning threshold, and ii) the height difference is within a given threshold. If more than one LiDAR-derived tree location match with a stem map location or vice versa, only the best one is used.

A scoring system was developed to match multiple LiDAR-derived tree locations with the most appropriate stem map location. Three increasing leaning $\left(5^{\circ}, 10^{\circ}\right.$, and $\left.15^{\circ}\right)$ and height difference $(10 \%, 20 \%$, and 30\%) threshold levels with decreasing scores $(100,70$, and 40) were considered (Table 2, Figure 5). A matrix with matching scores for all possible pairs of LiDARderived tree locations (rows) and stem map locations (columns) was then constructed. It was then processed by the Hungarian assignment algorithm (Kuhn, 1955) to produce the optimal matching assignment with the greatest overall matching scores.

Table 2. Leaning and height difference thresholds with associated scores considered for matching LiDAR-derived tree locations to stem map locations.

Leaning threshold Height difference threshold Score

\begin{tabular}{ccc}
$\left({ }^{0}\right)$ & $(\%)$ & \\
\hline 5 & 10 & 100 \\
10 & 20 & 70 \\
15 & 30 & 40 \\
$>$ & $>$ & 0 \\
\hline
\end{tabular}



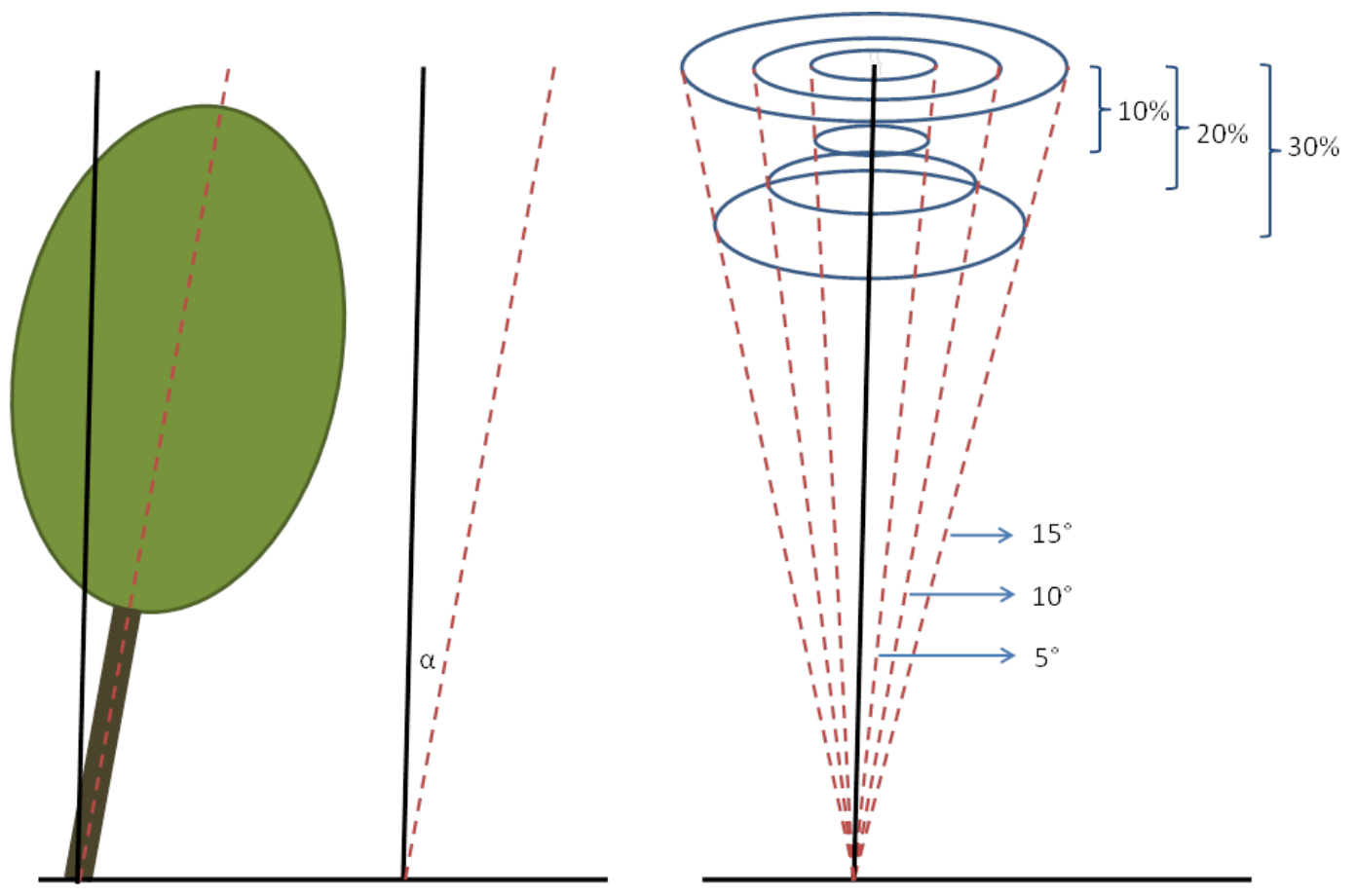

Figure 5. Calculation of leaning angle and distance difference used in the matching score system.

In the optimal assignment, a matched tree is an assigned pair of a LiDAR-derived tree location and a stem map location. An omission is a stem map location that remains unassigned $($ score $=0)$. A commission is an unassigned LiDAR-derived tree location. The number of matched trees (MT) is an indication of the segmentation quality. The number of omission errors $(\mathrm{OE})$ and commission errors (CE) indicate under- and over-segmentation, respectively. The accuracy of the approach was calculated in terms of recall $(\mathrm{Re})$, precision $(\mathrm{Pr})$ and F-score $(\mathrm{F})$ using the following equations (Manning et al., 2008):

$$
\begin{gathered}
R e=\frac{M T}{M T+O E} \\
P r=\frac{M T}{M T+C E} \\
F=2 \times \frac{R e \times P r}{R e+P r}
\end{gathered}
$$

Recall is a measure of the tree detection rate, precision is a measure of correctness of detected trees and the F-score indicates the overall accuracy taking omission and commission errors into account. 


\section{Results and Discussion}

After visually inspecting LiDAR ground points and intensity values, boards (and thus plot centers) were clearly identified for only 23 permanent plots, which were considered for the evaluation of the tree-segmentation approach. Although the location of the remaining plots could be estimated by triangulation to clearly visible objects on the ground and the LiDAR data (e.g., large trees, rock formations, vegetation gaps, road features), they were not considered in the analysis to avoid mismatching exact plot locations and thus obscuring comparisons between the tree-segmentation approach and the field-collected data. Plots were located on all aspect orientations and on slopes ranging from $10 \%$ to $70 \%$. An average of 13.2 trees were tallies per plot, with an average species diversity index (Shannon, 2001) of 1.47 (Table 3). The LiDAR point cloud over each plot included a 3.2-m (10 ft) buffer for capturing complete crowns of border trees.

Table 3. Summary plot level data collected from the 23 plots in the study area.

\begin{tabular}{|c|c|c|c|c|c|c|}
\hline Plot-Level Metric & & Min & $\operatorname{Max}$ & Average & Total & Percent of total \\
\hline Slope & $(\%)$ & 10 & 70 & 41 & & \\
\hline Aspect & 0 & 16 & 359 & 185 & & \\
\hline Tree count & & 6 & 27 & 13.2 & 303 & \\
\hline Dominant & & 0 & 3 & 0.6 & 14 & 4.6 \\
\hline Co-dominant & & 0 & 10 & 3.4 & 78 & 25.7 \\
\hline Intermediate & & 2 & 10 & 5.5 & 126 & 41.6 \\
\hline Overtopped & & 0 & 15 & 3.1 & 72 & 23.8 \\
\hline Dead & & 0 & 5 & 0.6 & 13 & 4.3 \\
\hline Species count & & 3 & 9 & 5.6 & 33 & \\
\hline $\begin{array}{l}\text { Shannon } \\
\text { diversity index }\end{array}$ & & 0.8 & 2.01 & 1.47 & & \\
\hline Median tree height & (m) & 13.0 & 24.7 & 18.3 & & \\
\hline $\begin{array}{l}\text { Interquartile range } \\
\text { of tree heights }\end{array}$ & (m) & 2.6 & 8.8 & 5.5 & & \\
\hline
\end{tabular}

The accuracy of the tree-segmentation approach on trees in the 23 plots is presented in Table 4. On average, the tree detection rate of the segmentation approach was $72 \%$, and $86 \%$ of detected trees were correctly detected. The overall accuracy in terms of the F-score was $77 \%$. Recall values ranged from $31 \%$ to $100 \%$ and precision values ranged from $50 \%$ to $100 \%$. In dense plots with a relatively large number of intermediate and overtopped trees, several trees 
were under-segmented resulting in relatively low recall values. For example, 6 of 19 and 0 of 11 intermediate and overtopped trees were detected in plots 4 and 11, respectively. However, all dominant and co-dominant trees in these two plots were detected. As expected, the three accuracy metrics were higher for dominant and co-dominant trees compared with intermediate and overtopped trees (Table 4). Recall increased to 94\% for larger trees and decreased to 62\% for smaller trees. Precision was more stable; it changed slightly about $1 \%$ from the overall $86 \%$, $87 \%$ for larger trees and $85 \%$ for smaller trees. When considering all trees, the tree-segmentation approach was able to detect $100 \%$ of dominant, $92 \%$ of co-dominant, $74 \%$ of intermediate, and $38 \%$ of overtopped trees in the 23 plots. In addition, the approach was able to detect $39 \%$ of dead trees (Table 4).

Table 4. Summary of accuracy results of the tree segmentation approach on the 23 plots.

\begin{tabular}{|c|c|c|c|c|c|c|c|c|c|c|c|c|c|c|c|c|}
\hline \multirow[b]{3}{*}{ Plot } & \multirow{2}{*}{\multicolumn{5}{|c|}{$\begin{array}{l}\text { Number of Lidar detected / } \\
\text { Field measured by tree class }\end{array}$}} & \multirow{2}{*}{\multicolumn{3}{|c|}{$\begin{array}{l}\text { Total number of } \\
\text { matches and errors }\end{array}$}} & \multirow{2}{*}{\multicolumn{2}{|c|}{$\begin{array}{c}\text { Overall } \\
\text { accuracy }(\%)\end{array}$}} & \multicolumn{6}{|c|}{ Accuracy by tree class group (\%) } \\
\hline & & & & & & & & & & & \multicolumn{3}{|c|}{$\mathrm{D} \& \mathrm{C}$} & \multicolumn{3}{|c|}{ I, O, \& Dead } \\
\hline & $\mathrm{D}^{1}$ & $\mathrm{C}^{2}$ & $\mathrm{I}^{3}$ & $\mathrm{O}^{4}$ & $\overline{\text { Dead }}$ & $\mathrm{MT}^{5}$ & $\mathrm{OE}^{6}$ & $\mathrm{CE}^{7}$ & $\operatorname{Re}^{8} \operatorname{Pr}^{9}$ & $\mathrm{~F}^{10}$ & $\operatorname{Re}$ & $\operatorname{Pr}$ & $\mathrm{F}$ & $\operatorname{Re}$ & $\operatorname{Pr}$ & $\bar{F}$ \\
\hline 1 & $0 / 0$ & $3 / 3$ & $6 / 10$ & $1 / 3$ & $0 / 0$ & 10 & 6 & 3 & 62.576 .9 & 69.0 & 100.0 & 75.0 & 85.7 & 53.8 & 77.8 & $\overline{63.6}$ \\
\hline 2 & $1 / 1$ & $3 / 3$ & $4 / 4$ & $2 / 6$ & $0 / 0$ & 10 & 4 & 1 & 71.490 .9 & 80.0 & 100.0 & 100.0 & 100.0 & 60.0 & 85.7 & 70.6 \\
\hline 3 & $0 / 0$ & $3 / 3$ & $4 / 4$ & $0 / 5$ & $0 / 1$ & 7 & 6 & 1 & $53.8 \quad 87.5$ & 66.6 & 100.0 & 75.0 & 85.7 & 40.0 & 100.0 & 57.1 \\
\hline 4 & $1 / 1$ & $4 / 4$ & $3 / 4$ & $3 / 15$ & $2 / 3$ & 13 & 14 & 0 & 48.1100 .0 & 65.0 & 100.0 & 100.0 & 100.0 & 36.4 & 100.0 & 53.3 \\
\hline 5 & $1 / 1$ & $4 / 4$ & 9/9 & $6 / 7$ & $0 / 0$ & 20 & 1 & 0 & 95.2100 .0 & 97.5 & 100.0 & 100.0 & 100.0 & 93.8 & 100.0 & 96.8 \\
\hline 6 & $2 / 2$ & $1 / 2$ & $3 / 3$ & $0 / 0$ & $0 / 0$ & 6 & 1 & 1 & 85.785 .7 & 85.7 & 75.0 & 100.0 & 85.7 & 100.0 & 75.0 & 85.7 \\
\hline 7 & $0 / 0$ & $9 / 10$ & $2 / 8$ & $0 / 3$ & $0 / 0$ & 11 & 10 & 4 & 52.473 .3 & 61.1 & 90.0 & 75.0 & 81.8 & 18.2 & 66.7 & 28.6 \\
\hline 8 & $1 / 1$ & $5 / 6$ & $5 / 8$ & $0 / 1$ & $0 / 0$ & 11 & 5 & 1 & 68.891 .7 & 78.6 & 85.7 & 85.7 & 85.7 & 55.6 & 100.0 & 71.4 \\
\hline 9 & $0 / 0$ & $2 / 2$ & $7 / 9$ & $2 / 3$ & $0 / 0$ & 11 & 3 & 1 & 78.691 .7 & 84.6 & 100.0 & 66.7 & 80.0 & 75.0 & 100.0 & 85.7 \\
\hline 10 & $0 / 0$ & $1 / 1$ & $2 / 2$ & $3 / 6$ & $1 / 5$ & 7 & 7 & 0 & 50.0100 .0 & 66.7 & 100.0 & 100.0 & 100.0 & 46.2 & 100.0 & 63.2 \\
\hline 11 & $1 / 1$ & $4 / 4$ & $0 / 8$ & $0 / 3$ & $0 / 0$ & 5 & 11 & 2 & 31.371 .4 & 43.5 & 100.0 & 71.4 & 83.3 & 00.0 & 00.0 & 00.0 \\
\hline 12 & $0 / 0$ & $4 / 4$ & $3 / 4$ & $2 / 3$ & $0 / 0$ & 9 & 2 & 4 & 81.869 .2 & 75.0 & 100.0 & 57.1 & 72.7 & 71.4 & 83.3 & 76.9 \\
\hline 13 & $0 / 0$ & $3 / 3$ & $7 / 7$ & $0 / 0$ & $0 / 0$ & 10 & 0 & 1 & 100.090 .9 & 95.2 & 100.0 & 100.0 & 100.0 & 100.0 & 87.5 & 93.3 \\
\hline 14 & $0 / 0$ & $2 / 2$ & $3 / 3$ & $1 / 1$ & $0 / 0$ & 6 & 0 & 0 & 100.0100 .01 & 00.0 & 100.0 & 100.0 & 100.0 & 100.0 & 100.0 & 100.0 \\
\hline 15 & $0 / 0$ & $9 / 9$ & $3 / 3$ & $0 / 0$ & $0 / 1$ & 12 & 1 & 2 & 92.385 .7 & 88.9 & 100.0 & 81.8 & 90.0 & 75.0 & 100.0 & 85.7 \\
\hline 16 & $1 / 1$ & $1 / 2$ & $5 / 8$ & $3 / 6$ & $0 / 0$ & 10 & 7 & 0 & 58.8100 .0 & 74.1 & 66.7 & 100.0 & 80.0 & 57.1 & 100.0 & 72.7 \\
\hline 17 & $0 / 0$ & $4 / 4$ & $6 / 6$ & $2 / 2$ & $1 / 1$ & 13 & 0 & 4 & 100.076 .5 & 86.7 & 100.0 & 66.7 & 80.0 & 100.0 & 81.8 & 90.0 \\
\hline 18 & $3 / 3$ & $0 / 0$ & $1 / 3$ & $0 / 0$ & $0 / 0$ & 4 & 2 & 1 & 66.780 .0 & 72.7 & 100.0 & 100.0 & 100.0 & 33.3 & 50.0 & 40.0 \\
\hline 19 & $2 / 2$ & $0 / 2$ & $2 / 4$ & $0 / 1$ & $0 / 0$ & 4 & 5 & 4 & 44.450 .0 & 47.0 & 50.0 & 66.7 & 57.1 & 40.0 & 40.0 & 40.0 \\
\hline 20 & $0 / 0$ & $2 / 2$ & $4 / 6$ & $0 / 0$ & $0 / 0$ & 6 & 2 & 2 & 75.075 .0 & 75.0 & 100.0 & 100.0 & 100.0 & 66.7 & 66.7 & 66.7 \\
\hline 21 & $0 / 0$ & $2 / 2$ & $4 / 5$ & $2 / 4$ & $1 / 1$ & 9 & 3 & 0 & 75.0100 .0 & 85.7 & 100.0 & 100.0 & 100.0 & 70.0 & 100.0 & 82.4 \\
\hline 22 & $1 / 1$ & $1 / 1$ & $6 / 6$ & $0 / 1$ & $0 / 1$ & 8 & 2 & 0 & 80.0100 .0 & 88.9 & 100.0 & 100.0 & 100.0 & 75.0 & 100.0 & 85.7 \\
\hline 23 & $0 / 0$ & $5 / 5$ & $2 / 2$ & $0 / 2$ & $0 / 0$ & 7 & 2 & 3 & $\begin{array}{ll}77.8 & 70.0 \\
\end{array}$ & 73.7 & 100.0 & 83.3 & 90.9 & 50.0 & 50.0 & 50.0 \\
\hline $\begin{array}{l}\text { Avera } \\
\text { detecti }\end{array}$ & $\begin{array}{l}4 / 12 \\
00 \%\end{array}$ & $\begin{array}{l}2 / 78 \\
2.3 \%\end{array}$ & $3 / 12$ & $\begin{array}{l}7 / 72 \\
7 \quad 507\end{array}$ & $\begin{array}{c}5 / 13 \\
38.6 \%\end{array}$ & $\begin{array}{r}206 / 30 \\
68.0 \%\end{array}$ & $4 / 30$ & $5 / 30$ & 71.785 .5 & 76.7 & 94.2 & 87.1 & 89.5 & 61.6 & 84.7 & 70.9 \\
\hline
\end{tabular}

${ }^{1}$ Dominant, ${ }^{2}$ Co-dominant, ${ }^{3}$ Intermediate, ${ }^{4}$ Overtopped

${ }^{5}$ Matched Trees, ${ }^{6}$ Omission Errors, ${ }^{7}$ Commission Errors, ${ }^{8}$ Recall, ${ }^{9}$ Precision, ${ }^{10}$ F-score

As an example, Figure 6 shows the results of the tree segmentation performance for plot 8, 14, 15, and 22. Empty areas close to plot boundaries represent crowns of non-matched trees 
outside the plots (apex is outside of the boundary), which were removed from the analysis. Omissions in these empty areas (i.e., lower right side of plot 8) are intermediate and overtopped trees likely below dominant trees outside the plot boundary. As the LiDAR point clouds include buffer areas, several matched tree crowns extend beyond the plot boundary. Many crowns do not look circular because of the dense canopies and the fact that the crowns may be undercover to some extent. Two commissions can be observed in plot 15 where nine co-dominant trees are growing tightly in a small area.
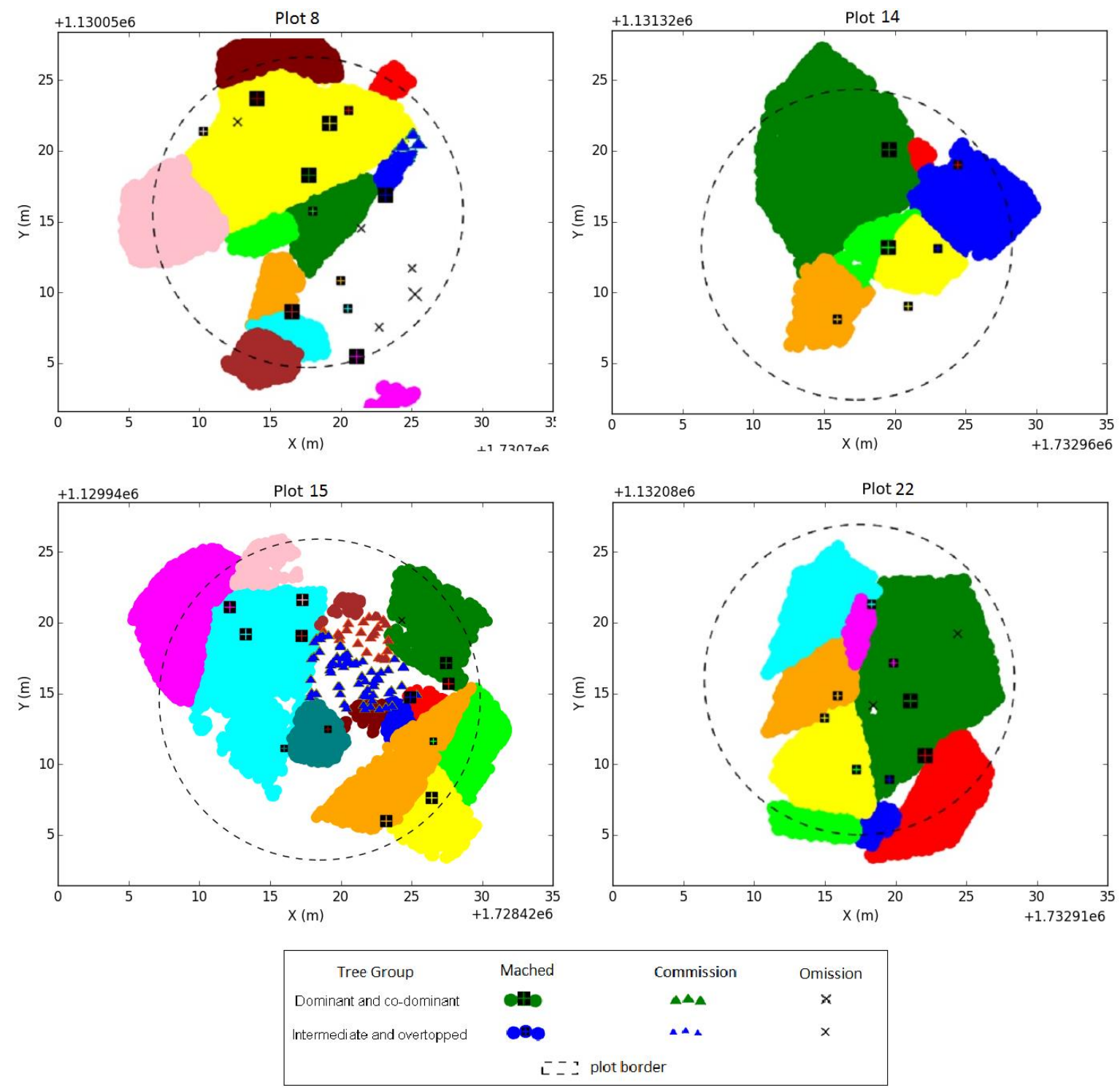

Figure 6. Aerial visualization of the tree segmentation results in four plots within the study area. Distinct colors represent matched tree crowns. 
We evaluated relationships between accuracy metrics for each tree group (precision, recall, and F-score) and plot level attributes, i.e., average terrain slope, tree density, species diversity index, percentage of dominant, co-dominant, intermediate, overtopped, and dead trees, as well as median and interquartile range of tree heights (IQRH). None of the relationships for dominant and co-dominant group of trees was statistically significant. For the smaller group of trees, we observed negative correlations between recall and IQRH $\left(\mathrm{P}=0.004, \mathrm{R}^{2}=0.33\right)$ and diversity index $\left(\mathrm{P}=0.03, \mathrm{R}^{2}=0.2\right)$. Similarly, there was a negative correlation between F-score and IQRH $\left(\mathrm{P}=0.03, \mathrm{R}^{2}=0.21\right)$. Also, a negative correlation between precision and percentage of dominant trees $\left(\mathrm{P}=0.02, \mathrm{R}^{2}=0.24\right)$ was observed. These correlations indicate that in multi-story plots with large dominant trees, intermediate and suppressed trees are more difficult to detect. As the tree segmentation approach considers only LSPs, dominant and co-dominant trees can be easily detected. On the other hand, the crowns of intermediate and overtopped trees are only partially visible from above and in some cases completely underneath large tree crowns, making them harder to be detected. As future work, we consider improving the approach by creating vertical canopy layers through examination of the height distribution of LiDAR point as suggested by Duncanson et al. (2014) and Wang et al (2008). The correlations we observed between accuracy metrics and plot level attributes are weak and insignificant specially for larger group of trees, which likely indicates that the accuracy of the tree-segmentation approach is not sensitive to differences in stand and terrain structures of the study area. This demonstrates the robustness of the approach and increases its potential applications.

Other tree-segmentation studies in closed-canopy deciduous forests have reported tree detection accuracies of about 50\% (Koch et al., 2006; Weinacker et al., 2004), 65\% (Jing et al., 2012), and 72\% (Hu et al., 2014) using similar evaluation metrics, which take both omissions and commissions into account. Vauhkonen et al. (2011) compared six different single tree detection methods on two deciduous forest sites. Performances were similar across sites; the average F-score of all methods was $57 \%$ where the maximum F-score was $64 \%$ and average recall and precision were $47 \%$ and $74 \%$, respectively. Also, Duncanson et al. (2014) used a multilayered crown delineation approach, which correctly identified $70 \%$ of dominant trees, $58 \%$ of co-dominant trees, $35 \%$ of intermediate trees, and $21 \%$ of overtopped trees in a deciduous forest. Tree-segmentation accuracies from these previous studies in deciduous forests are 
slightly lower than the accuracy from our novel approach, which is an indicator of potential applicability of our study to deciduous forests with complex vegetation conditions.

\section{Conclusions}

Developing automated approaches to obtain tree-level information over large forested areas is increasingly important for accurate assessment, monitoring and management. Most of existing methods are forest type specific and applied to conifer forests. In this study, we developed a generalized tree-segmentation approach that uses small foot print LiDAR data and applied it to natural deciduous forests with complex structures. A significant advantage of our novel approach is that it does not require a priori knowledge of tree shapes and sizes. The approach retrieves local information, crown steepness and height of the vegetation, and uses it on-the-fly to enhance crown delineation.

Using an improved evaluation method, results showed that our approach was able to detect $72 \%$ of trees, and $86 \%$ of detected trees were correctly identified, resulting in an overall accuracy of $77 \%$. Examining results by crown class, the approach detected $94 \%$ of dominant and co-dominant trees and $62 \%$ of intermediate and overtopped trees. Statistical analysis revealed similar accuracy levels across plots with different structures, which indicates the potential successful application of our approach to other forest types.

The main research challenge of the proposed tree segmentation approach was capturing heterogeneously shaped trees, and detecting intermediate and overtopped trees that may entirely be non-present within LSPs was not attempted explicitly. Future research will focus on incorporating multilayered segmentation method to improve detection of these smaller undercover trees. Our approach currently considers only LMs as the potential crown boundaries while other non-LM points might also represent boundaries, which in turn would result in omissions. Considering other points as potential crown boundaries (e.g., points showing distinct slope patterns on either side) can help to improve performance of the method. Although in general the number of omissions was larger than commissions, improving precision especially

for larger trees can also enhance overall accuracy. A post-processing step to identify oversegmentation, using a scoring mechanism to distinguish branch foliage from an entire tree crown, as suggested in (Hu et al., 2014; Véga et al., 2014; Wolf and Heipke, 2007) and then 
merging over-segmented crown sections back to form entire tree crowns can help to reduce commissions.

\section{Acknowledgements}

This work was supported by: 1) the Department of Forestry at the University of Kentucky and the McIntire-Stennis project KY009026 Accession 1001477, ii) the University of Kentucky Center for Computational Sciences, iii) the Kentucky Science and Engineering Foundation under the account number KSEF-3405-RDE-018, and iv) the National Science Foundation under Grant Number CCF-1215985.

\section{References}

Ackermann, F., 1999. Airborne laser scanning-present status and future expectations. ISPRS Journal of Photogrammetry and Remote Sensing 54, 64-67.

Alizadeh Khameneh, M.A., 2013. Tree detection and species identification using LiDAR data, School of Architecture and the Built Environment. Royal Institute of Technology, Stockholm, Sweden. Beucher, S., Lantuéjoul, C., 1979. Use of watersheds in contour detection, International workshop on image processing, real-time edge and motion detection.

Carpenter, S.B., Rumsey, R.L., 1976. Trees and shrubs of Robinson Forest Breathitt County, Kentucky. Castanea, 277-282.

Chen, Q., Baldocchi, D., Gong, P., Kelly, M., 2006. Isolating individual trees in a savanna woodland using small-footprint LiDAR data. Photogrammetric Engineering and Remote Sensing 72, 923-932.

Department of Forestry, 2007. Robinson Forest: a facility for research, teaching, and extension education. University of Kentucky.

Dougherty, E.R. and Lotufo, R.A., 2003. Hands-on morphological image processing. SPIE Press Bellingham.

Duncanson, L., Cook, B., Hurtt, G., Dubayah, R., 2014. An efficient, multi-layered crown delineation algorithm for mapping individual tree structure across multiple ecosystems. Remote Sensing of Environment 154, 378-386.

Falkowski, M.J., Smith, A.M., Hudak, A.T., Gessler, P.E., Vierling, L.A., Crookston, N.L., 2006. Automated estimation of individual conifer tree height and crown diameter via two-dimensional spatial wavelet analysis of lidar data. Canadian Journal of Remote Sensing 32, 153-161. 
Gougeon, F.A., 1995. A crown-following approach to the automatic delineation of individual tree crowns in high spatial resolution aerial images. Canadian journal of remote sensing 21, 274-284.

Heurich, M., 2008. Automatic recognition and measurement of single trees based on data from airborne laser scanning over the richly structured natural forests of the Bavarian Forest National Park. Forest Ecology and Management 255, 2416-2433.

Holmgren, J., Barth, A., Larsson, H., Olsson, H., 2012. Prediction of stem attributes by combining airborne laser scanning and measurements from harvesters. Holmgren, Johan, et al. "Prediction of stem attributes by combining airborne laser scanning and measurements from harvesters. Silva Fenn 46.2, 227-239.

Hu, B., Li, J., Jing, L., Judah, A., 2014. Improving the efficiency and accuracy of individual tree crown delineation from high-density LiDAR data. International Journal of Applied Earth Observation and Geoinformation 26, 145-155.

Hyyppä, J., Schardt, M., Haggrén, H., Koch, B., Lohr, U., Paananen, R., Scherrer, H., Luukkonen, H., Ziegler, M., Hyyppä, H., 2001. HIGH-SCAN: The first European-wide attempt to derive single-tree information from laserscanner data. The Photogrammetric Journal of Finland 17, 58-68.

Isenburg, M., 2011. LASTools - efficient tools for LiDAR processing. University of North Carolina - Chapel Hill.

Jing, L., Hu, B., Li, J., Noland, T., 2012. Automated delineation of individual tree crowns from LiDAR data by multi-scale analysis and segmentation. Photogrammetric engineering and remote sensing 78, 12751284.

Kaartinen, H., Hyyppä, J., Yu, X., Vastaranta, M., Hyyppä, H., Kukko, A., Holopainen, M., Heipke, C., Hirschmugl, M., Morsdorf, F., 2012. An international comparison of individual tree detection and extraction using airborne laser scanning. Remote Sensing 4, 950-974.

Kim, S., Hinckley, T., Briggs, D., 2009. Classifying tree species using structure and spectral data from LIDAR, ASPRS/MAPPS 2009 Specialty Conference.

Koch, B., Heyder, U., Weinacker, H., 2006. Detection of individual tree crowns in airborne LiDAR data. Photogrammetric Engineering \& Remote Sensing 72, 357-363.

Kuhn, H.W., 1955. The Hungarian method for the assignment problem. Naval Research Logistics Quarterly 2, 83-97.

Kwak, D.-A., Lee, W.-K., Lee, J.-H., Biging, G.S., Gong, P., 2007. Detection of individual trees and estimation of tree height using LiDAR data. Journal of Forest Research 12, 425-434. 
Leckie, D., Gougeon, F., Hill, D., Quinn, R., Armstrong, L., Shreenan, R., 2003. Combined high-density LiDAR and multispectral imagery for individual tree crown analysis. Canadian Journal of Remote Sensing 29, 633-649.

Li, W., Guo, Q., Jakubowski, M.K., Kelly, M., 2012. A new method for segmenting individual trees from the LiDAR point cloud. Photogrammetric Engineering \& Remote Sensing 78, 75-84.

Maltamo, M., Næsset, E., Vauhkonen, J., 2014. Forestry applications of airborne laser scanning. Concepts and case studies. Manag For Ecosys 27, 2014.

Manning, C.D., Raghavan, P., Schütze, H., 2008. Introduction to information retrieval. Cambridge university press Cambridge.

McGill, R., Tukey, J.W., Larsen, W.A., 1978. Variations of box plots. The American Statistician 32, 12-16. Morsdorf, F., Meier, E., Kötz, B., Itten, K.I., Dobbertin, M., Allgöwer, B., 2004. LiDAR-based geometric reconstruction of boreal type forest stands at single tree level for forest and wildland fire management. Remote Sensing of Environment 92, 353-362.

Overstreet, J., 1984. Robinson Forest inventory. Department of Forestry, University of Kentucky, Lexington, Kentucky.

Persson, A., Holmgren, J., Söderman, U., 2002. Detecting and measuring individual trees using an airborne laser scanner. Photogrammetric Engineering and Remote Sensing 68, 925-932.

Pitkänen, J., Maltamo, M., Hyyppä, J., Yu, X., 2004. Adaptive methods for individual tree detection on airborne laser based canopy height model. International Archives of Photogrammetry, Remote Sensing and Spatial Information Sciences 36, 187-191.

Popescu, S.C., Wynne, R.H., 2004. Seeing the trees in the forest. Photogrammetric Engineering \& Remote Sensing 70, 589-604.

Popescu, S.C., Wynne, R.H., Nelson, R.F., 2002. Estimating plot-level tree heights with LiDAR: local filtering with a canopy-height based variable window size. Computers and Electronics in Agriculture 37, 71-95.

Randolph, K.C., 2010. Equations relating compacted and uncompacted live crown ratio for common tree species in the South. Southern Journal of Applied Forestry 34, 118-123.

Schardt, M., Ziegler, M., Wimmer, A., Wack, R., Hyyppa, J., 2002. Assessment of forest parameters by means of laser scanning. ISPRS - The International Archives of the Photogrammetry, Remote Sensing and Spatial Information Sciences 34, 302-309.

Serra, J., 1986. Introduction to mathematical morphology. Computer Vision, Graphics, and Image Processing 35, 283-305. 
Shannon, C.E., 2001. A mathematical theory of communication. ACM SIGMOBILE Mobile Computing and Communications Review 5, 3-55.

Shao, G., Reynolds, K.M., 2006. Computer applications in sustainable forest management: Including perspectives on collaboration and integration. Springer Science \& Business Media.

Shiver, B.D., Borders, B.E., 1996. Sampling techniques for forest resource inventory. John Wiley and Sons.

Soille, P., 2003. Morphological Image Analysis: Principles and Applications. Springer-Verlag New York, Inc.

Terrasolid Ltd., 2012. TerraScan user's guide. Terrasolid Oy.

Thacker, N.A., Bromiley, P.A., 2001. The effects of a square root transform on a Poisson distributed quantity. Tina Memo 10, 2001.

Vauhkonen, J., Ene, L., Gupta, S., Heinzel, J., Holmgren, J., Pitkänen, J., Solberg, S., Wang, Y., Weinacker, H., Hauglin, K.M., 2011. Comparative testing of single-tree detection algorithms under different types of forest. Forestry, cpr051.

Véga, C., Durrieu, S., 2011. Multi-level filtering segmentation to measure individual tree parameters based on Lidar data: Application to a mountainous forest with heterogeneous stands. International Journal of Applied Earth Observation and Geoinformation 13, 646-656.

Véga, C., Hamrouni, A., El Mokhtari, S., Morel, J., Bock, J., Renaud, J.-P., Bouvier, M., Durrieu, S., 2014. PTrees: A point-based approach to forest tree extraction from lidar data. International Journal of Applied Earth Observation and Geoinformation 33, 98-108.

Vincent, L., Soille, P., 1991. Watersheds in digital spaces: an efficient algorithm based on immersion simulations. IEEE Transactions on Pattern Analysis and Machine Intelligence 13, 583-598.

Wang, Y., Weinacker, H., Koch, B., 2008. A lidar point cloud based procedure for vertical canopy structure analysis and 3D single tree modelling in forest. Sensors 8, 3938-3951.

Wehr, A., Lohr, U., 1999. Airborne laser scanning-an introduction and overview. ISPRS Journal of Photogrammetry and Remote Sensing 54, 68-82.

Weinacker, H., Koch, B., Heyder, U., Weinacker, R., 2004. Development of filtering, segmentation and modelling modules for lidar and multispectral data as a fundament of an automatic forest inventory system. ISPRS - International Archives of the Photogrammetry, Remote Sensing and Spatial Information Sciences 36, W2.

Wolf, B.-M., Heipke, C., 2007. Automatic extraction and delineation of single trees from remote sensing data. Machine Vision and Applications 18, 317-330. 
Appendix A: Expected slope of a spherical surface

The slope of a spherical surface in degrees ranges between 0-90 (Figure A.1). Assuming the LiDAR surface points are uniformly distributed along the horizontal dimension, the expected value of the angle $\alpha=\sin ^{-1} x$ is calculated as follows.

$$
\bar{\alpha}=\int_{0}^{1} \sin ^{-1} x \cdot d x=\frac{\pi}{2}-1=32.7^{\circ}
$$

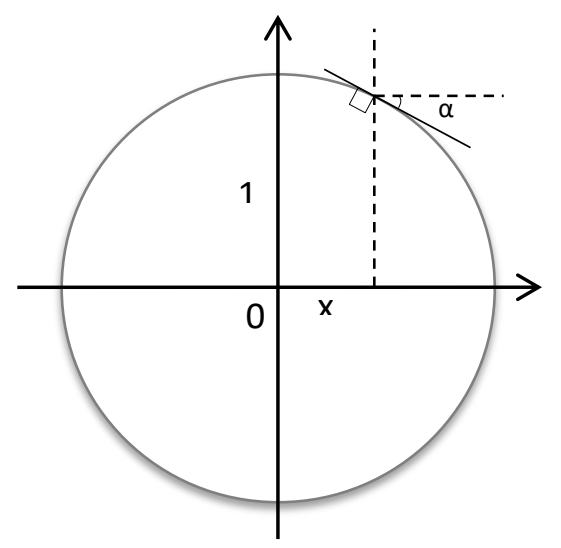

Figure A.1. The angle $\alpha$ representing the slope of the unit circle is a function of $x$. 